\title{
Localized Harmonic Characteristic Basis Functions for Multiscale Finite Element Methods
}

\author{
Leonardo A. Poveda* ${ }^{*}$ Sebastian Huepo ${ }^{\dagger} \quad$ Juan Galvis ${ }^{\ddagger}$ \\ Victor M. Calo ${ }^{\S}$
}

October 16, 2018

\section{Contents}

1 Introduction 2

2 Problem Setup 3

3 Asymptotic Expansion 4

4 An Expansion 7

5 Approximation with Localized Harmonic Characteristic Functions

6 Numerical Experiments 11

7 Conclusions 13

\begin{abstract}
We solve elliptic systems of equations posed on highly heterogeneous materials. Examples of this class of problems are composite structures and geological processes. We focus on a model problem which is a second-order elliptic equation

*Instituto de Matemática e Estatística, Universidade de São Paulo, Brazil, email: lpovedac@ime.usp.br

†'Departamento de Matemáticas, Universidad Nacional de Colombia, Bogotá D.C., Colombia, email: shuepobe@unal.edu.co

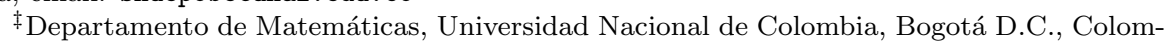
bia, email: jcgalvisa@unal.edu.co

$\S$ Center for Numerical Porous Media, Applied Mathematics. Computational Science and Earth Sciences \& Engineering, King Abdullah University of Science and Technology. Thuwal 23955-6900 Kingdom of Saudi Arabia, victor.calo@kaust.edu.sa
\end{abstract}


with discontinuous coefficients. These coefficients represent the conductivity of a composite material. We assume a background with low conductivity that contains inclusions with different thermal properties. Under this scenario we design a multiscale finite element method to efficiently approximate solutions. The method is based on an asymptotic expansions of the solution in terms of the ratio between the conductivities. The resulting method constructs (locally) finite element basis functions (one for each inclusion). These bases that generate the multiscale finite element space where the approximation of the solution is computed. Numerical experiments show the good performance of the proposed methodology.

keywords: Elliptic equation, asymptotic expansions, high-contrast coefficients, multiscale finite element method, harmonic characteristic function.

\section{Introduction}

Many physical and engineering applications naturally require multiscale solutions. This is specially true for problems related to metamaterials, composite materials, and porous media flows; see [2, 14, 15, 6, 20. The mathematical and numerical analyses for these problems are challenging since they are governed by elliptic equations with high-contrast coefficients $([11,3,7,1$,$) . For instance,$ in the modeling of composite materials, their conducting or elastic properties are modeled by discontinuous coefficients. The value of the coefficient can vary several orders of magnitude across discontinuities. Problems with these jumps are referred to as high-contrast. Similarly, the coefficient is denoted as a highcontrast coefficient. See for instance [1, 8, 5, 4].

We seek to understand how the high-contrast variations in the material properties affect the structure of the solution. In terms of the model, these variations appear in the coefficients of the differential equations. We expand our previous work [1, where we construct an asymptotic expansion to represent solutions. The asymptotic expansion is obtained in terms of the high contrast in the material properties. In this paper, we use this asymptotic expansion design numerical solutions for high-contrast problems. The asymptotic expansion helps us derive elegant numerical strategies and to understand the local behavior of the solution. In addition, the asymptotic expansion can be used to study functionals of solutions and describe their behavior with respect to the contrast or other important parameters. The asymptotic expansion in [1] uses globally supported harmonic extensions of subdomains indicator functions referred to as harmonic characteristic functions. We modify the construction presented in 1 to approximate the harmonic characteristic function in the local neighborhood of each inclusion. Thus, in order to make practical use of the expansion we avoid computing each characteristic function for the whole domain. This modification renders the method computationally tractable while the reduction in accuracy is not significant. For the case of dense distributions of inclusions, we observe numerically that the optimal size for the support of the basis functions is of the 
order of the representative distance between inclusions. We perform numerical tests that show the good performance of the proposed Multiscale Finite Element Method. We use a finite element method (FEM) and assume that there is a finemesh that completely resolves the geometrical configuration of the inclusions in the domain. That is, the fine-scale finite element formulation fully captures the solution behavior. To compute the linear system solution at this fine resolution is not practical and therefore, a multiscale finite element strategy is needed in order to compute a coarse-scale representation that captures relevant information of the targeted fine-scale solution. The coarse dimension in our simulations corresponds to the total number of inclusions. Nevertheless, a coarser scale may be needed for some applications. In this case it is possible to use the framework of the generalized finite element method to design and analyze a coarser scale for computations. For a detailed discussion, see [5] and references therein. In some more demanding applications an efficient iterative domain decomposition method could also be designed and analyzed for these problems. This is under investigation and will be presented elsewhere.

The problem of computing solutions of elliptic problems related to modern artificial materials such as dispersed and/or densely packed composite materials has been considered by some researchers recently. For instance in [19] the computation of effective properties of dispersed composite materials is considered. They use a classical multiscale finite element method. We recall that the application of the classical finite element method may lead to the precense of resonance errors due to the chosen local boundary conditions. See [10]. In [17, 16] the authors develop a finite element method based on a network approximation of the conductivity for particle composites.

The rest of the paper is organized as follows. In Section 2, we setup the problem. In Section 3, we summarize the asymptotic expansion procedure described in [1. We also introduce the definition of harmonic characteristic functions, which help us determine the individual terms of the expansion. In Section 4, we illustrate some aspects of the asymptotic expansion using some finite element computations. Section 5 constructs multiscale finite elements using the asymptotic expansion described in the previous sections. In particular, we approximate the leading term of the expansion with localized harmonic characteristic functions. We then apply this approximation to the case of dense high-contrast inclusions. In Section 6, we present some numerical experiments using the methods proposed. Finally, in Section 7 we draw some conclusions.

\section{Problem Setup}

We use the notation introduced in 1]. We consider a second order elliptic problems of the form,

$$
-\operatorname{div}(\kappa \nabla u)=f, \text { in } D,
$$

with Dirichlet data defined by $u=g$ on $\partial D$. Here $D$ is the disjoint union of a background domain $D_{0}$ and subdomains that represent the inclusions, i.e., $D=D_{0} \cup\left(\bigcup_{m=1}^{M} \bar{D}_{m}\right)$. We assume that $D_{1}, \ldots, D_{M}$ are connected polygonal 
domains (or domains with smooth boundaries). Additionally, we require that each inclusion $D_{m}, m=1, \ldots, M$ is compactly included in the open set $D \backslash$ $\bigcup_{\ell=1, \ell \neq m}^{M} \bar{D}_{\ell}$, i.e., $\bar{D}_{m} \subset D \backslash \bigcup_{\ell=1, \ell \neq m}^{M} \bar{D}_{\ell}$. Let $\kappa$ be defined by

$$
\kappa(x)= \begin{cases}\eta, & x \in D_{m}, \quad m=1, \ldots, M, \\ 1, & x \in D_{0}=D \backslash \bigcup_{m=1}^{M} \bar{D}_{m} .\end{cases}
$$

Following [1], we represent the solution by an asymptotic expansion in terms of the contrast $\eta$. The expansion reads,

$$
u_{\eta}=u_{0}+\frac{1}{\eta} u_{1}+\frac{1}{\eta^{2}} u_{2}+\cdots=\sum_{j=0}^{\infty} \eta^{-j} u_{j},
$$

with coefficients $\left\{u_{j}\right\}_{j=0}^{\infty} \subset H^{1}(D)$ and such that they satisfy the following Dirichlet boundary conditions,

$$
u_{0}=g \text { on } \partial D \quad \text { and } \quad u_{j}=0 \text { on } \partial D \text { for } j \geq 1 .
$$

\section{Asymptotic Expansion}

Now we compute the terms in the asymptotic expansion (3). For more details on the construction and related expansions we refer to [1, 18.

First, we introduce the harmonic characteristic functions. Let $\delta_{m \ell}$ represent the Kronecker delta, which is equal to 1 when $m=\ell$ and 0 otherwise. For each $m=1, \ldots, M$ we introduce the harmonic characteristic function of $D_{m}$, $\chi_{D_{m}} \in H_{0}^{1}(D)$, with the condition

$$
\chi_{D_{m}} \equiv \delta_{m \ell} \text { in } D_{\ell}, \text { for } \ell=1, \ldots, M,
$$

and which is equal to the harmonic extension of its boundary data to the interior of $D_{0}$. We then have,

$$
\begin{aligned}
\Delta \chi_{m} & =0, \text { in } D_{0}, \\
\chi_{m} & =0, \text { on } \partial D \text { and } \partial D_{\ell}, m \neq \ell, \ell=1,2, \ldots, M \\
\chi_{m} & =1, \text { on } \partial D_{m} .
\end{aligned}
$$

The function $u_{0}$ in $(3)$ can be explicitly written in term of the harmonic characteristic functions and a boundary corrector; see [1]. In fact, we can write,

$$
u_{0}=u_{0,0}+\sum_{m=1}^{M} c_{m}\left(u_{0}\right) \chi_{D_{m}}
$$

where $u_{0,0} \in H^{1}(D)$, for $m=1, \ldots, M$, and $u_{0,0}$ solves the following problem posed in the background $D_{0}$,

$$
\begin{aligned}
-\Delta u_{0,0} & =f, \text { in } D_{0}, \\
u_{0,0} & =g, \text { on } \partial D \\
u_{0,0} & =0, \text { on } \partial D_{m}, m=1, \ldots, M .
\end{aligned}
$$


$u_{0,0}$ is globally supported but it is forced to vanish in all interior inclusions boundaries. The constants in (7) solve an $M$ dimensional linear system. Let $\mathbf{c}=\left(c_{1}\left(u_{0}\right), \ldots, c_{M}\left(u_{0}\right)\right) \in \mathbb{R}^{M}$, then we have that

$$
\mathbf{A}_{\text {geom }} \mathbf{c}=\mathbf{b}
$$

where $\mathbf{A}_{\text {geom }}=\left[a_{m \ell}\right]$ and $\mathbf{b}=\left(b_{1}, \ldots, b_{M}\right) \in \mathbb{R}^{M}$ are defined by

$$
a_{m \ell}=\int_{D} \nabla \chi_{D_{m}} \cdot \nabla \chi_{D_{\ell}}
$$

and

$$
b_{\ell}=\int_{D} f \chi_{D_{\ell}}-\int_{D_{0}} \nabla u_{0,0} \cdot \nabla \chi_{D_{\ell}},
$$

respectively. $\sum_{m=1}^{M} c_{m}\left(u_{0}\right) \chi_{D_{m}}$ is the Galerkin projection of $u_{0}-u_{0,0}$ into the space $\operatorname{Span}\left\{\chi_{D_{m}}\right\}_{m=1}^{M}$.

Now for the sake of completeness, we briefly describe the next individual terms of the asymptotic expansion. We have for $j=1,2, \ldots$,

$$
u_{j}=\widetilde{u}_{j}+\sum_{m=1}^{M} c_{j, m} \chi_{D_{m}}
$$

where the function $\widetilde{u}_{j}$ is defined in three steps

1. Solve a Neumann problem in each inclusion with data from $u_{j-1}$ with $j=1,2, \ldots$ We describe the next terms in the asymptotic expansion. We have the restriction of $u_{j}$ to the subdomain $D_{m}$ with $m=1, \ldots, M$, that is

$$
u_{j}=\widetilde{u}_{j}+c_{j, m}, \quad \text { with } \int_{D_{m}} \widetilde{u}_{j}=0,
$$

and $\widetilde{u}_{j}$ satisfies the Neumann problem

$$
\int_{D_{m}} \nabla \widetilde{u}_{j} \cdot \nabla z=\int_{D_{m}} f z-\int_{\partial D_{m}} \nabla u_{j-1}^{(0)} \cdot n_{m} z, \text { for all } z \in H^{1}\left(D_{m}\right),
$$

for $m=1, \ldots, M$. From now on, we use the notation $w^{(0)}$, which means that the function $w$ is restricted to the domain $D_{0}$, that is $w^{(0)}=\left.w\right|_{D_{0}}$. The constants $c_{j, m}$ will be chosen suitably.

2. Solve a Dirichlet problem in the background $D_{0}$ with data $\widetilde{u}_{j}$ in each inclusion. For $j=1,2, \ldots$, we have that $u_{j}$ in $D_{m}, m=1, \ldots, M$, then we find $u_{j}^{(0)}$ in $D_{0}$ by solving the Dirichlet problem

$$
\begin{aligned}
\int_{D_{0}} \nabla u_{j}^{(0)} \cdot \nabla z & =0, & & \text { for all } z \in H_{0}^{1}\left(D_{0}\right) \\
u_{j}^{(0)} & =u_{j}\left(=\widetilde{u}_{j}+c_{j, m}\right), & & \text { on } \partial D_{m}, m=1, \ldots, M, \\
u_{j}^{(0)} & =0, & & \text { on } \partial D .
\end{aligned}
$$


Since $c_{j, m}$ are constants, we define their corresponding harmonic extension by $\sum_{m=1}^{M} c_{j, m} \chi_{D_{m}}$. So we rewrite

$$
u_{j}=\widetilde{u}_{j}+\sum_{m=1}^{M} c_{j, m} \chi_{D_{m}} .
$$

3. The $u_{j+1}$ in $D_{m}$ satisfy the following Neumann problem

$$
\int_{D_{m}} \nabla u_{j+1} \cdot \nabla z=-\int_{\partial D_{m}} \nabla u_{j}^{(0)} \cdot n_{0} z, \quad \text { for all } z \in H^{1}(D) .
$$

The compatibility condition is satisfied for $\ell=1, \ldots, M$, then

$$
\begin{aligned}
0=\int_{\partial D_{\ell}} \nabla u_{j+1} \cdot n_{\ell} & =-\int_{\partial D_{\ell}} \nabla u_{j}^{(0)} \cdot n_{0} \\
& =-\int_{D_{\ell}} \nabla\left(\widetilde{u}_{j}^{(0)}+\sum_{m=1}^{M} c_{j, m} \chi_{D_{m}}^{(0)}\right) \cdot n_{0} \\
& =-\int_{\partial D_{\ell}} \nabla \widetilde{u}_{j}^{(0)} \cdot n_{0}-\sum_{m=1}^{M} c_{j, m} \int_{\partial D_{m}} \nabla \chi_{D_{m}}^{(0)} \cdot n_{0} .
\end{aligned}
$$

A detailed description of the differential problems can be found in [1. The constants $\left\{c_{j, m}\right\}$ in 12 are computed solving a linear system similar to the one defined above in $(9)$. We have that $\mathbf{c}_{j}=\left(c_{j, 1}, \ldots, c_{j, M}\right)$ is the solution of the system

$$
\mathbf{A}_{\text {geom }} \mathbf{c}_{j}=\mathbf{y}_{j}
$$

where

$$
\mathbf{y}_{j}=\left(-\int_{D_{0}} \nabla \widetilde{u}_{j}^{(0)} \cdot \nabla \chi_{D_{1}}, \ldots,-\int_{D_{0}} \nabla \widetilde{u}_{j}^{(0)} \cdot \nabla \chi_{D_{M}}\right) .
$$

In [1, 18] the authors prove that the expansion (3) converges absolutely in $H^{1}(D)$ for $\eta$ sufficiently large.

Theorem 1. Consider the problem (1) with coefficient (2). The corresponding expansion (3) with boundary condition (4) converges absolutely in $H^{1}(D)$ for $\eta$ sufficiently large. Moreover, there exist positive constants $C$ and $C_{1}$ such that for every $\eta>C$, we have

$$
\left\|u-\sum_{j=0}^{J} \eta^{-j} u_{j}\right\|_{H^{1}(D)} \leq C_{1}\left(\|f\|_{H^{-1}(D)}+\|g\|_{H^{1 / 2}(\partial D)}\right) \sum_{j=J+1}^{\infty}\left(\frac{C}{\eta}\right)^{j},
$$

for $J \geq 0$. 


\section{An Expansion}

In this section we illustrate the expansion in two dimensions. We use MATLAB for the computations, see [9. In particular, a few terms are computed numerically using the finite element method, see for instance [13, 12. In particular, we solve the sequence of problems posed in the background subdomain and in the inclusions. Our main goal is to device efficient numerical approximations for $u_{0}$ (and then for $u_{\eta}$ by using Theorem 1).

We consider $D=B(0,1)$ the circle with center $(0,0)$ and radius 1 . We add 36 (identical) circular inclusions of radius 0.07. This is illustrated in the Figure 1a. Then, we numerically solve the problem

$$
\begin{cases}-\operatorname{div}\left(\kappa(x) \nabla u_{\eta}(x)\right)=1, & \text { in } D \\ u(x)=x_{1}+x_{2}^{2}, & \text { on } \partial D .\end{cases}
$$

Figure 1 shows that solution for $\eta=6$ against the computed $u_{0}$.

In Figure 2 we show the two parts of $u_{0}$ in (7), the combination of the harmonic characteristic functions $\sum_{m=1}^{M} c_{m}\left(u_{0}\right) \chi_{D_{m}}$ and the boundary corrector $u_{0,0}$. The results suggest that the boundary corrector $u_{0}$ decays fast away form the boundary $\partial D$.

In Figure 3 we show the second and third term of the expansions, $u_{1}$ and $u_{2}$. We also show the influence of $\eta$ on the convergence of the series in Table 1. As predicted by Theorem 1. as $\eta$ grows convergence of the series expansion is accelerated. For example, problem (15) with $\eta=10$ requires eight terms in the series to achieve a relative error of $10^{-8}$ while for $\eta=10^{4}$ requires only two terms.

\begin{tabular}{|c|c|c|c|c|c|c|c|c|c|c|}
\hline \hline$\eta$ & 3 & 6 & 10 & $10^{2}$ & $10^{3}$ & $10^{4}$ & $10^{5}$ & $10^{6}$ & $10^{7}$ & $10^{8}$ \\
\hline$\#$ & 25 & 11 & 8 & 4 & 3 & 2 & 2 & 2 & 1 & 1 \\
\hline \hline
\end{tabular}

Table 1: Number of terms needed to obtain a relative error of $10^{-8}$ for a given value of $\eta$.

\section{Approximation with Localized Harmonic Char- acteristic Functions}

In this section, we present a computable method based on the asymptotic expansion described in section 3 and the insights on the structure of the solution described in section 4 . We assume that $D$ is the union of a background and multiple inclusions that are homogeneously distributed. We only approximate of the leading term $u_{0}$. The remaining terms can be approximated similarly. We describe $u_{0}$ with localized harmonic characteristic functions. The computation of the harmonic characteristic functions is computationally expensive since 


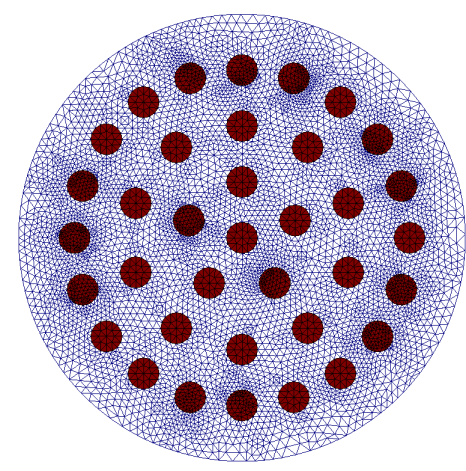

(a) Geometry and mesh.

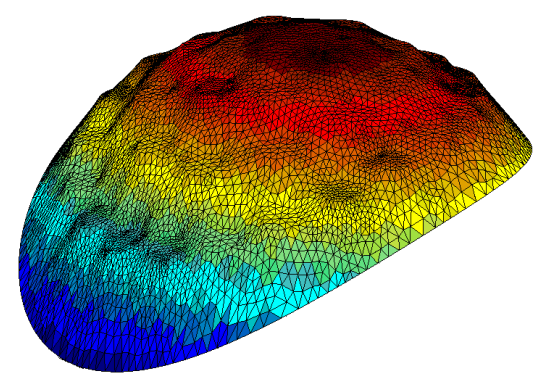

(b) Finite element solution of problem 15 with $\eta=6$.

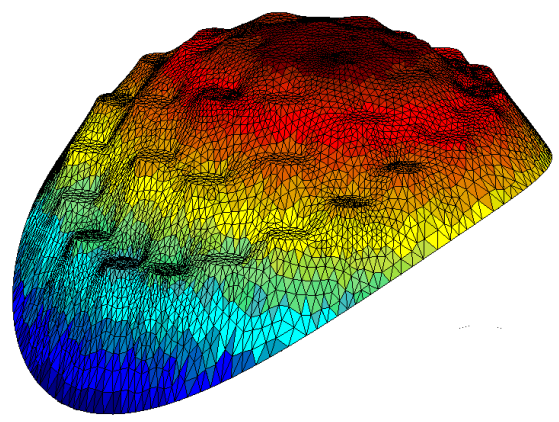

(c) Asymptotic solution $u_{0}$ in 7

Figure 1: Circular domain with 36 identical inclusions. Geometry, direct numerical simulation with finite elements, and asymptotic expansion.

these are fully global functions. That is, we approximate harmonic characteristics functions by solving a local problem (instead of a whole background 


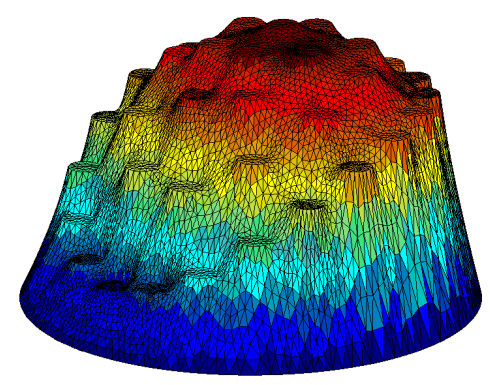

(a) $\sum_{m=1}^{M} c_{m}\left(u_{0}\right) \chi_{D_{m}}$

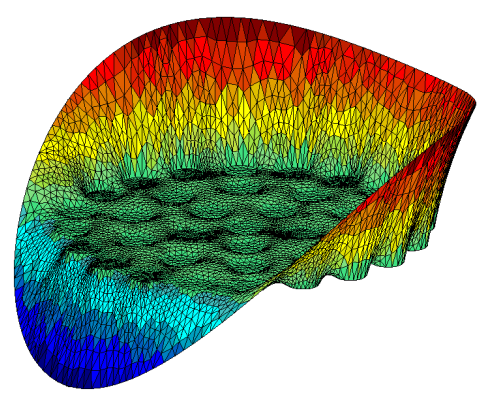

(b) $u_{0,0}$

Figure 2: Function $\sum_{m=1}^{M} c_{m}\left(u_{0}\right) \chi_{D_{m}}$ and $u_{0,0}$ for problem 15 with $\eta=6$. See (7).

problem). For instance, we pose a problem in a small neighborhood of each inclusion. The domain where the harmonic characteristic functions are computed is sketched in Figure 4. The domain marked in Figure 4 corresponds to the adopted neighborhood of the inclusion painted with green color. In this case, the approximated (or truncated) harmonic characteristic function is set to be zero on the boundary of the neighborhood of the selected inclusion.

The harmonic characteristic functions solve a background problem, which is global. To reduce the cost of computing the characteristic harmonic functions, we solve problem (15), but restrict it to a neighborhood of the corresponding inclusion. The exact characteristic functions are defined by (5). We define the neighborhood of the inclusion $D_{m}$ by

$$
D_{m, \delta}=\bar{D}_{m} \cup\left\{x \in D_{0}: d\left(x, D_{m}\right)<\delta\right\},
$$




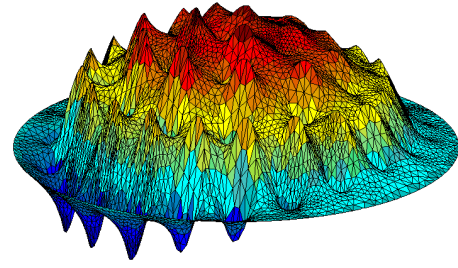

(a) $u_{1}$

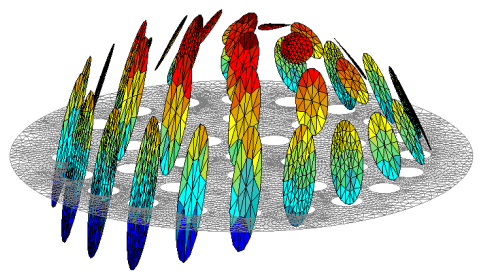

(c) $u_{1}$ restricted to the inclusions

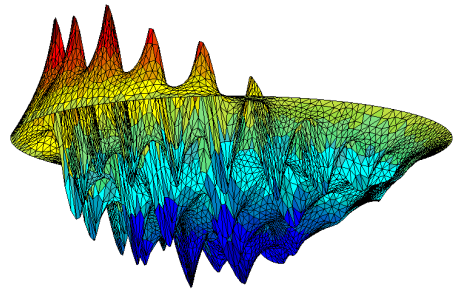

(b) $u_{2}$

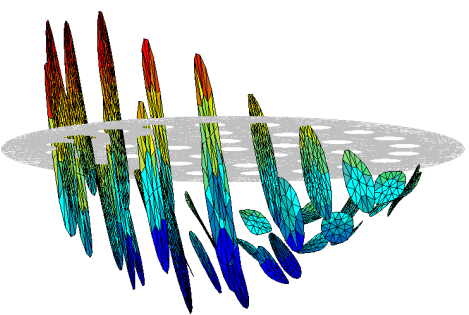

(d) $u_{2}$ restricted to the inclusions

Figure 3: Top: Functions $u_{1}$ and $u_{2}$ for the problem 15 with $\eta=6$. Bottom: Functions $u_{1}$ and $u_{2}$ restricted to the inclusions for the problem (15) with $\eta=6$.

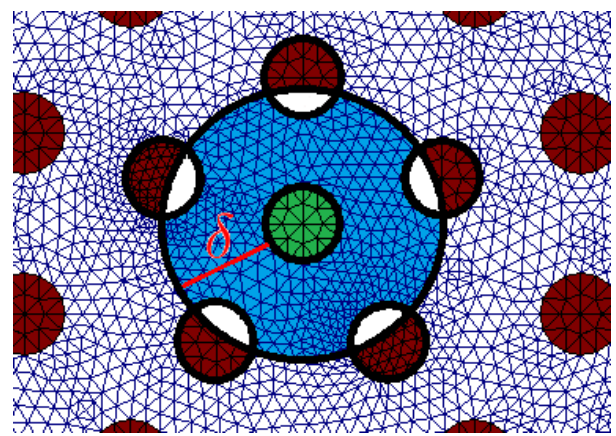

Figure 4: Illustration of $\delta$-neighborhood of an inclusion. The selected inclusion is green. The $\delta$-neighborhood of this inclusion is given in blue color, while preserving the truncated harmonic characteristics function. We highlight with white color the other inclusions that are within the $\delta$-neighborhood of the selected inclusion.

and approximate the characteristic function for the $\delta$-neighborhood solving

$$
\begin{aligned}
\Delta \chi_{m}^{\delta} & =0, \text { in } D_{m, \delta}, \\
\chi_{m}^{\delta} & =0, \text { on } \partial D_{m, \delta} \text { and } \partial D_{\ell} \cap D_{m, \delta} \text { for } \ell \neq m, \\
\chi_{m}^{\delta} & =1, \text { on } \partial D_{m} .
\end{aligned}
$$


The exact expression for $u_{0}$ is given by

$$
u_{0}=u_{0,0}+\sum_{m=1}^{M} c_{m} \chi_{D_{m}}=u_{0,0}+u_{c}
$$

where we have introduced $u_{c}=\sum_{m=1}^{M} c_{m}\left(u_{0}\right) \chi_{D_{m}}$. The matrix problem for $u_{c}$ with globally supported basis was given in (9). We now define $u_{0}^{\delta}$, the mulsticale approximation of $u_{0}$, using a similar expression which is given by

$$
u_{0}^{\delta}=u_{0,0}^{\delta}+\sum_{m=1}^{M} c_{m}^{\delta} \chi_{D_{m}}^{\delta}=u_{0,0}^{\delta}+u_{c}^{\delta},
$$

where $u_{c}^{\delta}=\sum_{m=1}^{M} c_{m}^{\delta} \chi_{D_{m}}^{\delta}$ with each $c_{m}^{\delta}$ computed similarly to $c_{m}$ using an alternative matrix problem with basis $\chi_{D_{m}}^{\delta}$ instead of $\chi_{D_{m}}$. This system is given by

$$
\mathbf{A}^{\delta} \mathbf{c}^{\delta}=\mathbf{b}^{\delta} .
$$

with $\mathbf{A}^{\delta}=\left[a_{m \ell}^{\delta}\right]$, with $a_{m \ell}^{\delta}=\int_{D_{m}} \nabla \chi_{m}^{\delta} \cdot \nabla \chi_{\ell}^{\delta}, \mathbf{c}=\left[c_{0}^{\delta}\left(u_{0}\right), \ldots, c_{M}^{\delta}\left(u_{0}\right)\right]$ and $\mathbf{b}^{\delta}=\left[b_{\ell}^{\delta}\right]=\int_{D} f \chi_{D_{\ell}}^{\delta}$.

We also introduced the $u_{0,0}^{\delta}$, that is an approximation to the boundary corrector $u_{0,0}$, that solves the problem

$$
\begin{aligned}
-\Delta u_{0,0}^{\delta} & =f, \text { in } D_{0}^{\delta}, \\
u_{0,0}^{\delta} & =g, \text { on } \partial D \\
u_{0,0}^{\delta} & =0, \text { on } \partial D_{0}^{\delta} \cap D,
\end{aligned}
$$

where $D_{0}^{\delta}$ is the subdomain within a distance $\delta$ from the boundary $\partial D$,

$$
D_{0}^{\delta}=\{x \in D: d(x, \partial D)<\delta\} .
$$

\section{$6 \quad$ Numerical Experiments}

To show the effectiveness of the numerical methodology described in section 5 . we first consider the problem configuration used in Section 4 . See Figure 1. We study the expansion which localizes the harmonic characteristic functions. We first compare the global and the localized harmonic characteristic functions. In Figure 5 we plot the global characteristic function (left picture) corresponding to a randomly selected inclusion. By construction this harmonic characteristic function is zero at the boundary of all other inclusions which imply a fast decay away from the inclusion. In addition, we plot the difference between the localized characteristic function in (16) and the characteristic function in (5); see Figure 5b. Here we use $\delta=0.3$ and observe that the maximum value of this absolute difference is 0.016 . 


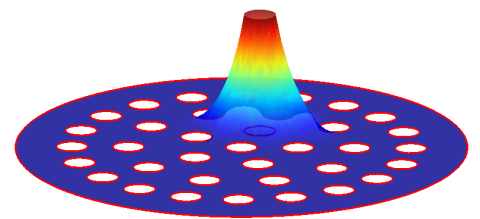

(a) Illustration of the Global characteristic function for the problem 15 .

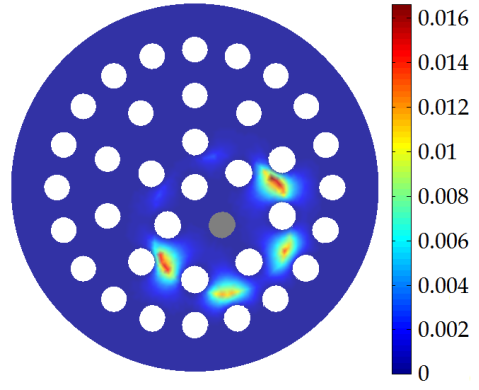

(b) Difference between the global characteristic function (5) and the localized characteristic function 16 .

Figure 5: Global versus localized characteristic functions. Here we use $\delta=0.3$ and obtained maximum error of 0.016 .

\begin{tabular}{|c|c|c|c|}
\hline \hline$\delta$ & $e\left(u_{0}-u_{0}^{\delta}\right)$ & $e\left(u_{0,0}-u_{0,0}^{\delta}\right)$ & $e\left(u_{c}-u_{c}^{\delta}\right)$ \\
\hline \hline 0.001 & 0.830673 & 0.999907 & 0.555113 \\
0.05 & 0.530459 & 0.768135 & 0.549068 \\
0.10 & 0.336229 & 0.639191 & 0.512751 \\
0.20 & 0.081500 & 0.261912 & 0.216649 \\
0.30 & 0.044613 & 0.088706 & 0.048173 \\
0.40 & 0.041061 & 0.047743 & 0.007886 \\
0.50 & 0.033781 & 0.034508 & 0.001225 \\
0.60 & 0.029269 & 0.029362 & 0.000174 \\
0.70 & 0.020881 & 0.020888 & 0.000021 \\
0.80 & 0.012772 & 0.012773 & 0.000003 \\
0.90 & 0.006172 & 0.006172 & 0.000000 \\
\hline \hline
\end{tabular}

Table 2: Relative error in the approximation of $u_{0}$ by using locally computed basis functions and truncated boundary condition effect. Here $u_{0}=u_{0,0}+u_{c}$ where $u_{c}$ is combination of harmonic characteristic functions and $u_{0}^{\delta}=u_{0,0}^{\delta}+u_{c}^{\delta}$ is computed by solving $u_{0,0}^{\delta}$ on a $\delta$-strip of the boundary $\partial D$ and the basis functions on a $\delta$-strip of the boundary of each inclusion.

For further comparison, we introduce the relative $H^{1}$ error from $u_{0}$ in 17 to its approximation $u_{0}^{\delta}$ in 18 . This is given by

$$
e\left(u_{0}-u_{0}^{\delta}\right)=\frac{\left\|u_{0}-u_{0}^{\delta}\right\|_{H^{1}}}{\left\|u_{0}\right\|_{H^{1}}} .
$$

Analogously, the relative $H^{1}$ error of the approximation of $u_{0,0}$ is given by

$$
e\left(u_{0,0}-u_{0,0}^{\delta}\right)=\frac{\left\|u_{0,0}-u_{0,0}^{\delta}\right\|_{H^{1}}}{\left\|u_{0,0}\right\|_{H^{1}}} .
$$

The error $e\left(u_{c}-u_{c}^{\delta}\right)$ is defined in a similar way. According to Theorem 1 the 


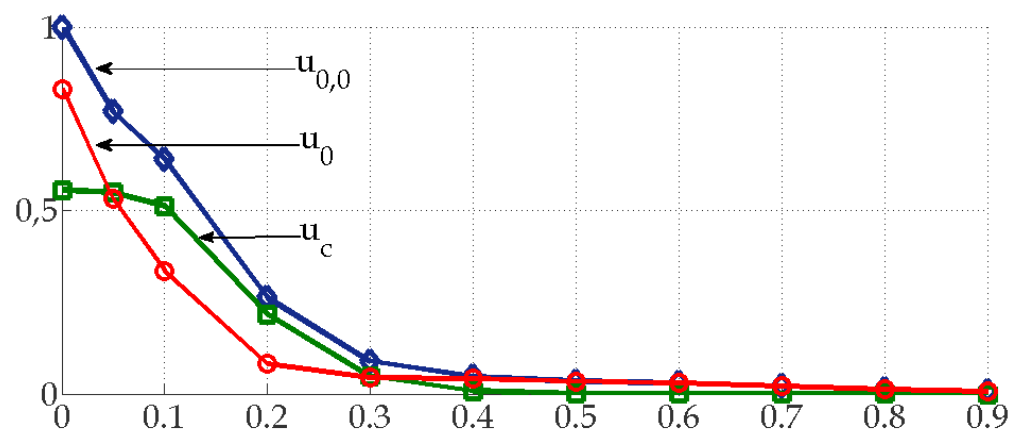

Figure 6: Relative error in the approximation of $u_{0}$ given by Table 2

error between the exact solution of problem (1) with coefficient (2) and $u_{0}$ in (17) is of order $\eta^{-1}$.

Table 2 and Figure 6 show the relative errors of the multiscale method. In Table 2, we observe that as the neighborhood size grows, the error is reduced. For instance, for $\delta=0.2$ the error between the exact solution $u_{0}$ and the truncated solution $u_{0}^{\delta}$ is $8 \%$. By selecting $\delta=0.3$ we obtain a relative error of the order of $4 \%$. Numerically we observe that an optimal value for $\delta$ is the smallest distance that includes one layer of inclusions away from the selected one. Therefore, this approximation is more efficient for densely packed inclusions.

We now consider an additional geometrical configuration of inclusions. We consider $D=(0,1)$, the circle with center $(0,0)$ and radius 1 , and 60 (identical) circular inclusions of radius 0.07 . We consider the problem,

$$
\begin{cases}-\operatorname{div}\left(\kappa(x) \nabla u_{\eta}(x)\right)=1, & \text { in } D \\ u(x)=x_{1}+x_{2}^{2}, & \text { on } \partial D,\end{cases}
$$

In the Figure 7 we illustrate the geometry for problem (19). Table 3 summarizes similar results to those described above.

The problem setup and simulation of the localized harmonic characteristic functions proceeds as described in Section 5. In the present setup the inclusions are clustered more tightly. This induces a faster decay of the characteristic harmonic function for each individual inclusion, see Figure 8 . Thus, as expected in Table 3 we observe a reduction in the relative error when compared to Table 2 for a fixed value of $\delta$. For example, $\delta=0.2$ induces a relative error of $1 \%$ on $u_{0}^{\delta}$ and of $2 \%$ for $u_{0,0}^{\delta}$ for the geometry show in the Figure 7 while this value of $\delta$ induces errors of $8 \%$ and $26 \%$ for these two variables for the geometry shown in Figure 1a.

\section{Conclusions}

We consider the solution of elliptic problems modeling properties of composite materials. Using an expansion in terms of the properties ratio presented 


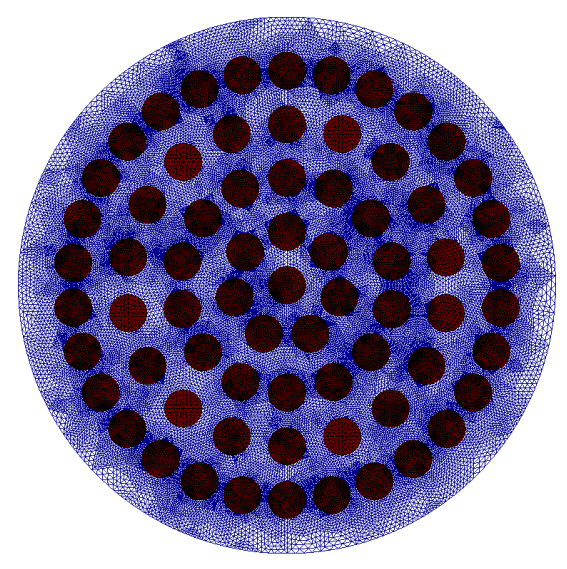

Figure 7: Geometry for the problem $\sqrt{19}$.

\begin{tabular}{|c|c|c|c|}
\hline \hline$\delta$ & $e\left(u_{0}-u_{0}^{\delta}\right)$ & $e\left(u_{00}-u_{0,0}^{\delta}\right)$ & $e\left(u_{c}-u_{c}^{\delta}\right)$ \\
\hline \hline 0.001 & 0.912746 & 0.999972 & 0.408063 \\
0.05 & 0.369838 & 0.549332 & 0.399472 \\
0.10 & 0.181871 & 0.351184 & 0.258946 \\
0.20 & 0.013781 & 0.020172 & 0.011061 \\
0.30 & 0.013332 & 0.013433 & 0.000737 \\
0.40 & 0.010394 & 0.010396 & 0.000057 \\
0.50 & 0.009228 & 0.009228 & 0.000004 \\
0.60 & 0.006102 & 0.006102 & 0.000000 \\
0.70 & 0.005561 & 0.005561 & 0.000000 \\
0.80 & 0.002239 & 0.002239 & 0.000000 \\
0.90 & 0.001724 & 0.001724 & 0.000000 \\
\hline \hline
\end{tabular}

Table 3: Relative error in the approximation of $u_{0}$ when using locally computed basis functions and a truncated boundary conditions. $e(w)=\|w\|_{H^{1}} /\left\|u_{0}\right\|_{H^{1}}$. Here $u_{0}=u_{0,0}+u_{c}$ where $u_{c}$ is combination of harmonic characteristic functions and $u_{0}^{\delta}=u_{0,0}^{\delta}+u_{c}^{\delta}$ is computed by solving $u_{0,0}^{\delta}$ on a $\delta$-strip of the boundary $\partial D$ and the basis functions on a $\delta$-strip of the boundary of each inclusion.

in [1], we design a multiscale method to approximate solutions. We develop procedures that effectively and accurately compute the first few terms in the expansion. In particular, we compute the asymptotic limit which is an approximation of order $\eta^{-1}$ to the solution (where $\eta$ represent the ratio between lowest and highest material property values). The expansion in [1] is written in terms of the harmonic characteristic functions that are globally supported functions, one for each inclusion. The main idea we propose is to approximate the harmonic characteristic functions by solving local problems around each inclusion. We use numerical examples to compute the asymptotic limit $u_{0}$ with the localized harmonic characteristic functions. The analysis of the truncation 


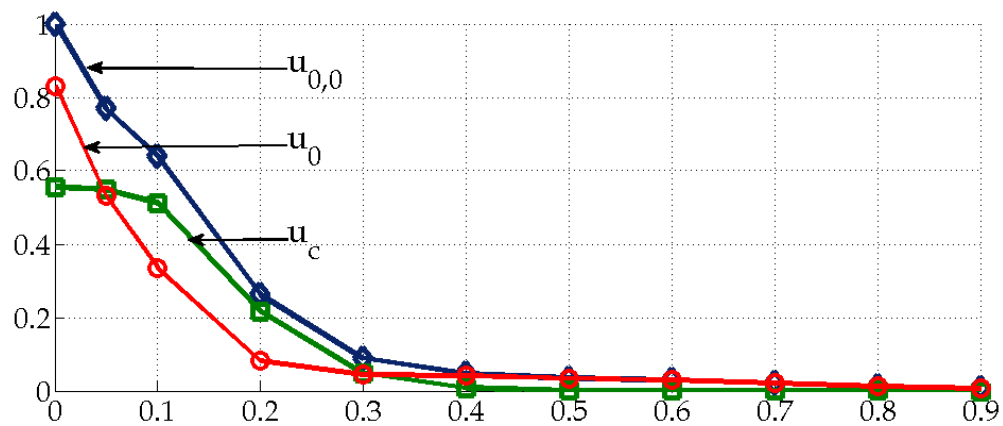

Figure 8: Relative error in the approximation of $u_{0}$ given by Table 3

error depends on decay properties of the harmonic characteristic functions and is under current investigation. This method can be used in several important engineering applications with heterogeneous coefficients such as complex flow in porous media and complex modern materials.

\section{References}

[1] V. M. Calo, Y. Efendiev, and J. Galvis, Asymptotic expansions for high-contrast elliptic equations, Math. Models Methods Appl. Sci, 24 (2014), pp. 465-494.

[2] Y. Chen And R. Lipton, Multiscale methods for engineering double negative metamaterials, Photonics and Nanostructures-Fundamentals and Applications, 11 (2013), pp. 442-452.

[3] Z. Chen And T. Hou, A mixed multiscale finite element method for elliptic problems with oscillating coefficients, Mathematics of Computation, 72 (2003), pp. 541-576.

[4] Y. Efendiev and J. Galvis, Coarse-grid multiscale model reduction techniques for flows in heterogeneous media and applications, in Numerical Analysis of Multiscale Problems, Springer, 2012, pp. 97-125.

[5] Y. Efendiev, J. Galvis, and T. Y. Hou, Generalized multiscale finite element methods, Journal of Computational Physics, 251 (2013), pp. 116135 .

[6] M. Epov, V. Terekhov, M. Nizovtsev, E. Shurina, N. Itkina, And E. UKOLOV, Effective thermal conductivity of dispersed materials with contrast inclusions, High Temperature, 53 (2015), pp. 45-50.

[7] J. Galvis and Y. Efendiev, Domain decomposition preconditioners for multiscale flows in high-contrast media, Multiscale Modeling \& Simulation, 8 (2010), pp. 1461-1483. 
[8] J. Galvis And S. Ki KAng, Spectral multiscale finite element for nonlinear flows in highly heterogeneous media: A reduced basis approach, Journal of Computational and Applied Mathematics, 260 (2014), pp. 494-508.

[9] M. U. GuIDE, The mathworks, Inc., Natick, MA, 5 (1998), p. 333.

[10] U. Hetmaniuk, Multiscale finite element methods. Theory and applications, SIAM Rev., 53 (2011), pp. 389-390.

[11] T. Y. Hou AND X.-H. Wu, A multiscale finite element method for elliptic problems in composite materials and porous media, Journal of computational physics, 134 (1997), pp. 169-189.

[12] T. J. Hughes, The finite element method: linear static and dynamic finite element analysis, Dover Publications, Inc., Mineola, NY, 2012.

[13] C. Johnson, Numerical solution of partial differential equations by the finite element method, Dover Publications, Inc., Mineola, NY, 2009. Reprint of the 1987 edition.

[14] J. LI, Finite element study of the Lorentz model in metamaterials, Computer Methods in Applied Mechanics and Engineering, 200 (2011), pp. 626637.

[15] O. Ozgun and M. Kuzuoglu, Software metamaterials: Transformation media based multiscale techniques for computational electromagnetics, Journal of Computational Physics, 236 (2013), pp. 203-219.

[16] D. Peterseim and C. Carstensen, Finite element network approximation of conductivity in particle composites, Numerische Mathematik, pp. 125 .

[17] _ Finite element network approximation of conductivity in particle composites, Numerische Mathematik, 124 (2013), pp. 73-97.

[18] L. A. Poveda, S. Huepo, V. M. Calo, and J. Galvis, Asymptotic expansions for high-contrast linear elasticity, Journal of Computational and Applied Mathematics, 295 (2016), pp. 25-34.

[19] D. Wen And Y. Ding, Effective thermal conductivity of aqueous suspensions of carbon nanotubes (carbon nanotube nanofluids), Journal of Thermophysics and Heat Transfer, 18 (2004), pp. 481-485.

[20] K. Zhou, L. M. Keer, Q. J. Wang, X. Ai, K. Sawamiphakdi, P. Glaws, M. PAIRE, AND F. CHE, Interaction of multiple inhomogeneous inclusions beneath a surface, Computer methods in applied mechanics and engineering, 217 (2012), pp. 25-33. 